\title{
Usos y significados de los objetos en niños mexicanos
}

Pedro Palacios Salas, ${ }^{1}$ Cintia Rodríguez Garrido, ${ }^{2}$ Cecilia Méndez Sánchez, ${ }^{1}$ Alicia Edith Hermosillo de la Torre ${ }^{1}$ y Miguel Ángel Sahagún Padilla ${ }^{1}$

Existen, al menos, dos maneras claramente diferenciadas de concebir cómo los niños conocen los objetos. La primera de ellas, y con mayor tradición en la psicología, se caracteriza por plantear 1) que los niños, por sus propios medios, llegan a conocer las propiedades físicas de los objetos y 2) que el mundo material es asemiótico, que carece de reglas de uso y significados culturales. Esta visión asemiótica del mundo material, reducida a sus aspectos físicos, está en la base de distintos planteamientos psicológicos. Para Piaget (1936/1985, 1945/1976), a los niños les basta utilizar sus esquemas sensoriomotores como instrumentos para conocer cualquier objeto, sin importar de qué objeto en particular se trate. La vasta diversidad de esquemas de acción sensoriomotora que encontramos en Piaget ha sido reducida al esquema de explorar por algunos teóricos: explorando el objeto es como los niños descubren

Departamento de Psicología, Centro de Ciencias Sociales y Humanidades. Universidad Autónoma de Aguascalientes.

2 Departamento de Psicología Evolutiva y Educación, Facultad de Psicología. Universidad Autónoma de Madrid. 
sus propiedades físicas (Bjorklund y Gardiner, 2011), sus affordances naturales (Tomasello, 1999) e incluso sus affordances intencionales (Rakoczy, Tomasello y Striano, 2005; Tomasello y Rakoczy, 2003). Por último, para Leslie $(1987,1994)$ las representaciones primarias innatas les permiten a los niños conocer el mundo de forma directa, literal, precisa, transparente y semántica.

La segunda manera de concebir cómo los niños conocen los objetos enfatiza 1) en que los objetos, además de sus atributos físicos, tienen reglas de uso y significados culturales que se definen por las funciones que tienen en la vida cotidiana y 2) que el adulto y el propio objeto juegan un papel protagónico en el origen y comprensión de los usos y significados asignados a los objetos (Moro y Rodríguez, 2005; Palacios, 2009; Palacios y Rodríguez, 2006; Palacios, Rodríguez, Méndez-Sánchez y Hermosillo-de la Torre, 2012, 2013; Rodríguez, 2012; Rodríguez y Moro, 1999). Desde la pragmática del objeto, el mundo material no está separado de las normas de uso o de sus propiedades funcionales, por lo que el conocimiento de los objetos incluye los aspectos semióticos sociales de los significados normativos de uso de los mismos. Gracias a que las tazas se usan como tazas es que llegan a ser signo de dicho uso y para que esto ocurra se requiere que se usen como tales. A su vez, las reglas de los usos convencionales o canónicos de los objetos sirven como significados de primer grado sobre los que se asientan los significados simbólicos o de segundo grado (Palacios y Rodríguez, 2015; Rodríguez, Palacios, Cárdenas y Yuste, 2014). Con el símbolo o uso simbólico se evoca el significado ausente del uso canónico del objeto. En suma, los significados asignados a los objetos dependen de sus usos y el niño requiere que los adultos les asignen usos y significados canónicos y simbólicos a los objetos mientras se comunican e interactúan conjuntamente con los objetos para que acaben apropiándose de dichos conocimientos o maneras de significar el mundo. El objetivo del presente trabajo es mostrar los cambios en las prácticas de uso de los objetos que las díadas mexicanas realizaron con los objetos en el transcurso de los 9 a los 18 meses de edad de los niños. 


\section{Método}

\section{Participantes}

En el presente estudio participaron seis niños mexicanos (tres niños y tres niñas) con uno de sus respectivos padres y fueron seleccionados de manera no probabilística. Los padres fueron contactados por medio de las directoras de Centros de Desarrollo Infantil en la ciudad de Aguascalientes, Ags. Los niños tenían 9 meses al inicio del estudio. La edad promedio de los padres fue de 31.66 años. Las familias participantes pertenecen a un nivel socioeconómico medio.

\section{Material}

Se utilizaron diez objetos familiares para los participantes: un bote de crema vacío, un caballo de plástico, una caja de cartón con una cuerda atada al frente, un cepillo de dientes para niño, una cuchara de madera, un encendedor de plástico convencional, un muñeco de tela, una piedra pequeña ovalada, un teléfono celular de juguete que reproduce sonidos musicales y voces al pulsar sus teclas y un trapo.

Para la filmación, usamos una cámara de video digital.

\section{Procedimiento}

Los participantes fueron observados en cuatro ocasiones a lo largo de 9 meses en sus contextos familiares. La primera observación se realizó cuando los niños/as tenían 9 meses. La segunda filmación tuvo lugar a los 12 meses, la tercera a los 15 y la cuarta a los 18 meses de edad. Todas las observaciones se realizaron en el hogar de los participantes en un contexto comunicativo triádico semiestructurado (adulto-niño-objeto). Pedimos a los adultos que se sentaran junto a su hijo/a, depositamos los 10 objetos frente a ellos a una distancia que permitiera cogerlos libremente y les dimos la siguiente instrucción: "Interactúe con su hijo/a como suele hacerlo normalmente y trate de olvidar que lo 
estamos grabando". Enseguida comenzamos a grabarlos durante 10 minutos.

Posteriormente, hicimos la toma de protocolo de las interacciones triádicas adulto-niño-objeto, segundo a segundo, a partir de las filmaciones.

Una vez realizados los protocolos, el siguiente paso fue segmentarlos en secuencias o episodios de uso de los objetos. Para realizar el corte en secuencias tomamos como criterio general la triadicidad, esto es, que el adulto y el niño mantuvieran la atención o acción simultánea en torno al mismo objeto por lo menos durante 5 segundos. La secuencia inicia cuando uno de los participantes usa un objeto y el otro participante mira o participa en la actividad. La secuencia finaliza cuando uno de los participantes rompe la atención convergente, inicia otro tipo de práctica o uso con el mismo objeto o cuando cambia de objeto.

La codificación se realizó usando las categorías semióticas de usos no canónicos y usos canónicos descritas por Rodríguez y Moro (1999) y Moro y Rodríguez (2005), así como la de usos simbólicos descrita por Palacios (2009).

Usos no canónicos. Usos indiferenciados e inespecíficos que los niños les dan a los objetos. Estos usos se corresponden desde una lectura semiótica con el iconismo de Peirce, ya que la relación entre representamen y su objeto inmediato no se apoya en otras relaciones previas.

Usos canónicos. Usos sujetos a reglas públicas y culturales. Los objetos son usados de acuerdo con sus usos cotidianos y públicos. Se corresponden con el concepto de símbolo de Peirce, donde la relación entre representamen y su objeto inmediato es producto de una regla o ley.

Usos simbólicos. Usos de los objetos que representan algo ausente. Estos usos se apoyan en las reglas de los usos canónicos. Tales reglas pueden ser aplicadas al mismo objeto en contextos o escenarios distintos, a otros objetos o a ningún objeto, donde la regla del uso canónico de un objeto es evocada en la ausencia de algún objeto.

Finalmente, hicimos un análisis estadístico descriptivo de las frecuencias y duraciones de las secuencias. 


\section{Resultados}

Porcentaje del tiempo total empleado

por las díadas en los usos de los objetos

A los 9 meses, los participantes invirtieron mayor porcentaje de tiempo en usar los objetos de forma no canónica (29.88\%) comparado con el tiempo invertido en los usos canónicos y simbólicos, cuyos porcentajes fueron muy similares (cerca de 13\%). En cambio, a partir de los 12 meses, los porcentajes de tiempo que los participantes invirtieron en las secuencias canónicas y simbólicas fueron mayores que el de los usos no canónicos. El porcentaje de la duración total de las secuencias canónicas disminuyó progresivamente tendiendo a cero conforme la edad de los niños. El porcentaje de la duración total de las secuencias canónicas aumentó a los 12 meses y luego disminuyó ligeramente a los 15 y 18 meses, pero sin ser menor que el de los 9 meses, manteniéndose más o menos constante a lo largo del estudio. Finalmente, el porcentaje de la duración total de los usos simbólicos fue el que mayor incremento tuvo conforme la edad de los niños, alcanzando casi $60 \%$ a los 18 meses.

Número total y duración media de las secuencias de los usos de los objetos

La frecuencia y duración media de las secuencias no canónicas siempre fueron en decremento conforme la edad de los niños, siendo mayores a los 9 meses. En relación con los usos canónicos, la frecuencia incrementó a los 12 meses, comparada con los 9 meses. Posteriormente, a los 15 y 18 meses, hay una disminución progresiva en comparación con la de los 12 meses, pero sin llegar a estar por debajo de la de los 9 meses. La duración media de las secuencias canónicas se mantiene más o menos constante desde los 9 hasta los 18 meses. La frecuencia total de las secuencias simbólicas incrementó a los 12 meses en comparación con los 9 meses y, a partir de los 12 meses, se mantiene relativamente constante. La duración media de las secuen- 
cias simbólicas tuvo un incremento progresivo y ligero a los 12 y 15 meses y un incremento mayor a los 18 meses (casi el doble), en relación con los 9 meses.

\section{Discusión}

El tiempo que los participantes invierten en los distintos usos de los objetos se relaciona con el desarrollo cognitivo alcanzado por los niños/as. Comienzan usando los objetos de acuerdo con lo que las características físicas de los objetos les permiten: manipularlos, chuparlos, golpearlos, etcétera. A partir de los 12 meses, los usos no canónicos iniciales de los objetos por parte de los niños van siendo reemplazados por usos culturales basados en reglas, como los usos canónicos y simbólicos. Estos datos cuestionan la concepción prevaleciente en torno a la manera en que los niños llegan a conocer los objetos, así como el concepto mismo de objeto y la idea de "la realidad literal" evidente para los niños. Asimismo, los adultos juegan un papel muy importante en la transmisión de estas formas culturales de usos de los objetos (Moro y Rodríguez, 2005; Palacios, 2009; Palacios y Rodríguez, 2006, 2015; Palacios et al., 2012; Rodríguez, 2006; Rodríguez y Moro, 1999), donde los contextos triádicos, con su carácter asimétrico y dialéctico, se constituyen en el escenario de los procesos de desarrollo y educativos (Palacios et al., 2013).

\section{Referencias}

Bjorklund, D.F., y Gardiner, A.K. (2011). Object Play and Tool Use: Developmental and Evolutionary Perspectives. En A.D. Pellegrini (Ed.), The Oxford Handbook of Development of Play (153-171). EUA: Oxford University Press.

Leslie, A.M. (1987). Pretense and Representation: The Origins of "Theory of Mind". Psychological Review, 94(4), 412-426. Leslie, A.M. (1994). Pretending and Believing: Issues in the Theory of ToMM. Cognition, 50, 211-238. 
Moro, C. y Rodríguez, C. (2005). L'objet et la construction de son usage chez le bébé. Une approche sémiotique du développement préverbal. Bern-New York: Peter Lang.

Palacios, P. (2009). Origen de los usos simbólicos de los objetos en los niños en contextos de comunicación e interacción triádicos (Tesis doctoral no publicada). Facultad de Psicología, Universidad Autónoma de Madrid, Madrid, España.

Palacios, P. y Rodríguez, C. (2006). Los símbolos en los contextos de interacción tríadica. En P. Palacios (Ed.), Memoria. Primer Congreso Internacional de Psicología del Desarrollo. Primer Encuentro de Egresados (pp. 1-9). Aguascalientes, México: UAA.

Palacios, P. y Rodríguez, C. (2015). The Development of Symbolic Uses of Objects in Infants in a Triadic Context: A Pragmatic and Semiotic Perspective. Infant and Child Development. Published online in Wiley Online Library. DOI: 10.1002/icd.1873.

Palacios, P., Rodríguez, C., Méndez-Sánchez, C. y Hermosillo-de la Torre, A.E. (2012). Participación del adulto en la construcción de los usos convencionales de los objetos. En R. Díaz-Loving, S. Rivera-Aragón e I. Reyes-Lagunes (Comp.), Aportaciones actuales de la psicología social. Volumen 1 (6-28). Monterrey: UANL.

Palacios, P., Rodríguez, C., Méndez-Sánchez, C. y Hermosillo de la Torre, A. E. (2013). Contextos comunicativos e interactivos tríadicos: la unidad básica de la educación. En P. Palacios, M.C. Farfán, E. Navarrete y S.C. Miramontes (Comp.), Psicología educativa: reflexión, práctica e intervención (1440). Toluca, México: UAEM.

Piaget, J. (1985). El nacimiento de la inteligencia en el niño (trad.

P. Bordonaba). Barcelona: Crítica (Trabajo original publicado en 1936).

Piaget, J. (1976). La formation du symbole chez l'enfant. Neuchatel: Delachaux \& Niestlé. (Trabajo original publicado en 1945).

Rakoczy, H., Tomasello, M., y Striano, T. (2005). How Children Turn Objects into Symbols: A Cultural Learning Account. 
En L. Namy (Ed.), Symbol Use and Symbolic Representation (69-97). Mahwah-London: LEA.

Rodríguez, C. (2006). Del ritmo al símbolo. Los signos en el nacimiento de la inteligencia. Barcelona: Horsori.

Rodríguez, C. (2012). The Functional Permanence of the Object: A Product of Consensus. En E. Martí y C. Rodríguez (Eds.), After Piaget (123-150). New Brunswick, NJ/London: Transactions Publishers.

Rodríguez, C. y Moro, C. (1999). El mágico número tres. Cuando los niños aún no hablan. Barcelona: Paidós.

Rodríguez, C., Palacios, P., Cárdenas, K. y Yuste, N. (2014). Les symboles: des formes de second ou de troisiéme sens? En C. Moro y N. Muller-Mirza (Eds.), Psychologie du développement, sémiotique et cultura (99-116). Villeneuve d'Ascq: Presses Universitaires du Septentrion.

Tomasello, M. (1999). The Cultural Origins of Human Cognition. Cambridge: Harvard University Press.

Tomasello, M. y Rakoczy, H. (2003). What Makes Human Cognition Unique? From Individual to Shared to Collective Intentionality. Mind and Language, 18(2), 121-147. 